\title{
General Psychiatry Risk factors of hyperprolactinemia induced by risperidone and olanzapine and their correlations with plasma glucose and lipids
}

\author{
Sidi He, ${ }^{1}$ Wen Juan Yu, ${ }^{1}$ Xiaoliang Wang, ${ }^{1}$ Lei Zhang, ${ }^{1}$ Nan Zhao, ${ }^{2}$ Guanjun Li, ${ }^{1}$ \\ Yi Feng Shen, ${ }^{1}$ Huafang $\mathrm{Li}^{1,3,4}$
}

To cite: He S, Yu WJ, Wang $X$, et al. Risk factors of hyperprolactinemia induced by risperidone and olanzapine and their correlations with plasma glucose and lipids. General Psychiatry 2020;33:e100206. doi:10.1136/ gpsych-2020-100206

Received 10 February 2020 Revised 27 April 2020 Accepted 01 June 2020

\section{Check for updates}

\section{Author(s) (or their} employer(s)) 2020. Re-use permitted under CC BY-NC. No commercial re-use. See rights and permissions. Published by BMJ.

${ }^{1}$ Shanghai Mental Health Center, Shanghai Jiao Tong University School of Medicine, Shanghai,

China

${ }^{2}$ Shanghai Pudong New Area Mental Health Center, Shanghai, China

${ }^{3}$ Clinical Research Center, Shanghai Jiao Tong University School of Medicine, Shanghai, China

${ }^{4}$ Shanghai Key Laboratory of Psychotic Disorders, Shanghai, China

Correspondence to

Dr Huafang Li; Ihlh_5@163.com

\section{ABSTRACT}

Background Hyperprolactinemia is a common adverse reaction in patients with schizophrenia who take antipsychotic drugs; it often leads to treatment noncompliance in patients and has an adverse effect on their prognosis.

Aims This study aimed to explore the risk factors of elevated prolactin (PRL) caused by risperidone (RIS) and olanzapine (OLZ) and the relationship between PRL and fasting plasma glucose and lipids.

Methods Patients with schizophrenia were divided into two groups: 264 patients who were taking RIS and 175 patients who were taking OLZ. These two groups were further divided according to serum PRL levels: an elevated PRL group (>30 $\mathrm{ng} / \mathrm{mL}$ ) and a normal PRL group (PRL $\leq 30 \mathrm{ng} / \mathrm{mL}$ ). The demographics, medication dosage, fasting plasma glucose, total cholesterol and triglycerides were compared in the two groups. Logistic regression analysis was performed to explore the risk factors of elevated PRL levels.

Results Compared with the OLZ group, the RIS group had a greater number of patients with elevated PRL (155/264 vs 58/175). Either the RIS or the OLZ group, the proportion of elevated PRL was greater in female patients (RIS: $\chi^{2}=6.76, p=0.009 ; 0$ LZ: $\chi^{2}=12.98, p<0.001$ ) and with higher doses of the related drugs (RIS: $U=-3.73$, $\mathrm{p}<0.001$; OLZ: $U=-2.31, \mathrm{p}=0.021)$. In patients taking RIS, the elevated PRL subgroup took the drug for a longer period $(U=-2.76, p=0.006)$ and had lower triglyceride levels $(U=2.76, p=0.006)$. In patients taking $0 L Z$, the elevated PRL subgroup had lower fasting plasma glucose levels $(U=2.29, p=0.022)$. Logistic regression analysis showed that gender, dose and fasting glucose levels were significantly associated with elevated PRL levels (RIS: $p=0.001$, OLZ: $p<0.001$; RIS: $p<0.001 ;$ OLZ: $p=0.003$; RIS: $p=0.020,0 L Z: p=0.001$, respectively).

Conclusion Compared with OLZ, RIS had a greater effect on PRL in patients with schizophrenia, and in patients with schizophrenia taking RIS or OLZ, gender and dose were significantly correlated with the PRL value. Moreover, the plasma glucose level of the group with elevated PRL was lower than that of the group with normal PRL. The results also showed that high serum PRL may be associated with a favourable glucose metabolic profile in patients with schizophrenia taking RIS or OLZ. Further studies are warranted to confirm this association.

\section{Trial registration number NCT02640911}

\section{INTRODUCTION}

Schizophrenia is a chronic mental illness with a prevalence of about $1 \%$ and usually requires long-term treatment with antipsychotics. ${ }^{1}$ Elevated prolactin (PRL) levels are one of the common adverse reactions that patients experience after taking antipsychotic drugs, which lead to a reduction in drug compliance and subsequently affect prognosis. ${ }^{2}$

PRL is a polypeptide hormone secreted by the lactotropic cells in the anterior pituitary. ${ }^{3}$ The factors that influence the effect of different levels of PRL caused by antipsychotic drugs include age, gender, type of antipsychotic drug, dosage of medication and length of time for which medication is taken. ${ }^{4}$ The incidence of hyperprolactinemia in menopausal women is lower than that in women of childbearing age. ${ }^{5}$ Among male patients, age has less effect on elevated PRL levels. ${ }^{6}$ Women tend to have higher levels of PRL than men and may be associated with higher levels of oestrogen. ${ }^{7}$ In addition, the level of PRL is also related to medication dosage, with higher doses of antipsychotics leading to higher levels of PRL. ${ }^{8}$ Previous studies in elderly patients with schizophrenia have found that after reducing medication dose, the $\mathrm{D}_{2} / \mathrm{D}_{3}$ receptor occupancy rate decreases and PRL levels significantly decrease. ${ }^{9}$

PRL is a multifunctional hormone. Studies have shown that PRL is related to the growth of pancreatic cells and the function of insulin. ${ }^{10}$ Animal studies have shown that PRL levels have a direct stimulating effect on insulin in non-pregnant rodents and can induce insulin production. ${ }^{11}$ In addition, studies have found that maternal PRL increases concurrently to insulin during the second half of pregnancy and stimulates $\beta$-cell proliferation 


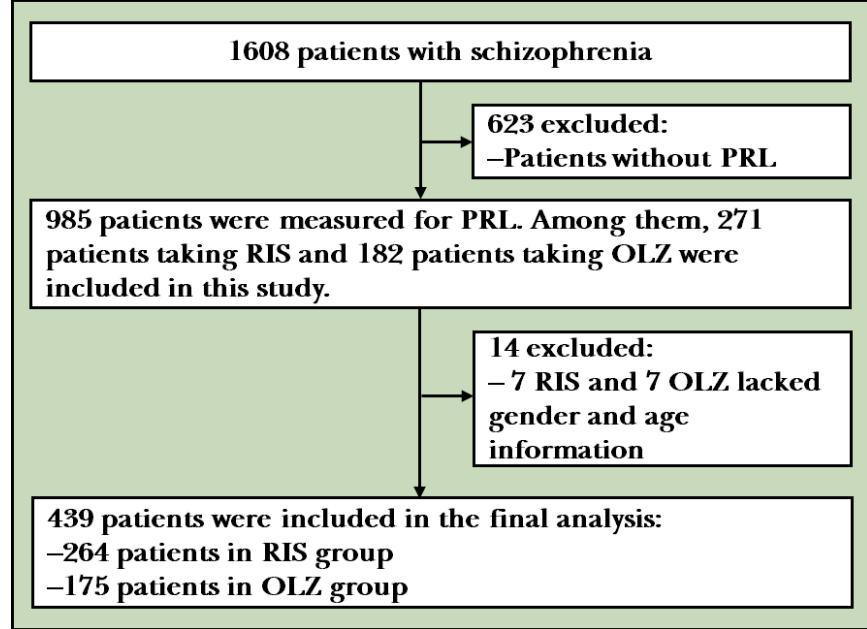

Figure 1 Flowchart of the study. OLZ, olanzapine; PRL, prolactin; RIS, risperidone.

and insulin secretion. ${ }^{12}$ Current studies suggest that the glucose metabolic regulation effect of PRL is not limited to the period of pregnancy. ${ }^{11}$ Moreover, studies have discovered that human adipose tissue produces PRL and also expresses PRL receptors, which highlights the role of PRL as a cytokine involved in adipose tissue function. ${ }^{14}$ PRL can downregulate lipoprotein lipase and fatty acid synthesis, which inhibits lipogenesis, and regulate the bioactivities of adipokines such as adiponectin, interleukin-6 and leptin. ${ }^{1516}$ Studies have reported that elevated PRL is associated with lower levels of glucose and lipids, as well as higher insulin sensitivity. ${ }^{17}$ At present, it is unclear whether PRL level is correlated with plasma glucose and lipids in patients with schizophrenia taking antipsychotic drugs as part of their treatment.

Risperidone (RIS) and olanzapine (OLZ) are widely used in the treatment of schizophrenia, and their therapeutic effects have been recognised in long-term clinical practice. Both RIS and OLZ exert antipsychotic effects by blocking dopamine $\mathrm{D}_{2}$ and serotonin $5-\mathrm{HT}_{2 \mathrm{~A}}$ receptors. The dopamine $\mathrm{D}_{2}$ receptor occupancy rate is correlated with adverse drug reactions. ${ }^{18}$ The meta-analyses have shown that OLZ has less effect on PRL levels in adolescent patients than RIS. ${ }^{19}$ In addition, a meta-analysis of World Psychiatry in 2019 included 16 studies in a subgroup analysis of elevated PRL levels. The results indicated that OLZ had a significantly more favourable effect on PRL levels than RIS. ${ }^{20}$ The purpose of this study was to investigate the risk factors for elevated PRL levels in patients with schizophrenia taking RIS and OLZ and to explore the relationship between PRL and plasma glucose and lipids levels.

\section{METHODS}

\section{Participants}

The sample was derived from a real-world, multicentre clinical trial-an observational safety and related factors study on atypical antipsychotics long-term treatment in
Chinese patients with schizophrenia (An Observational Safety and Related Factors Study on Atypical Antipsychotics Long-term Treatment in Chinese Patients with Schizophrenia, SALT-C). The study was organised by the Shanghai Mental Health Center, reviewed and approved by its Ethics Committee and assigned to the International Clinical Trials Registry Platform number NCT02640911. The multicentre study included 13 hospitals, Shanghai Mental Health Center, Peking University Sixth Hospital, Beijing Anding Hospital Capital Medical University, Guangzhou Psychiatric Hospital, West China Hospital, Sichuan University, The Second Xiangya Hospital of Central South University, First Affiliated Hospital of Kunming Medical University, First Affiliated Hospital of Xian Jiaotong University, Shanghai Luwan Area Mental Health Center, Shanghai Baoshan Area Mental Health Center, Shanghai Pudong New Area Mental Health Center, Shanghai Minhang Area Mental Health Center and Shanghai Jiading Area Mental Health Center. All patients in this study met the following inclusion criteria: (1) patients must have currently met or met in the past the Diagnostic and Statistical Manual of Mental Disorders, Fourth Edition, criteria for schizophrenia. The diagnosis was made independently by at least two attending psychiatrists, and the diagnosis had to be consistent; (2) patients were 18 years old or above; (3) patients were taking quetiapine/olanzapine/risperidone/aripiprazole/ziprasidone/paliperidone/amisulpride/perospirone/clozapine; and (4) all patients and their legal guardians must have signed a written informed consent form. Exclusion criteria were as follows: (1) participation in other studies; (2) a serious tendency or history of suicidal thoughts, severe excitement, injury or uncooperative treatment; and (3) the presence of comorbid physical illness, alcohol or drug abuse or dependence. As shown in figure 1 , a total of 1608 patients were included in the SALT-C study, and 985 were measured for PRL. Among them, 271 patients taking RIS and 182 patients taking OLZ were included in this study. Owing to a lack of demographic data, seven RIS and seven OLZ cases were excluded. In the final analysis, 439 patients were included: 264 patients in the RIS group and 175 patients in the OLZ group.

\section{Data collection}

General demographic information included gender, age and body mass index. Disease-related information included illness duration, age of onset, medication time, doses of RIS and OLZ, fasting plasma glucose, total cholesterol and triglycerides. For determining the PRL level, venous blood $(2 \mathrm{~mL})$ was collected on an empty stomach in the early morning. After centrifugation, the serum was collected and serum PRL was detected by radioimmunoassay. This research was based on cross-sectional baseline data and combined with literature reports. Elevated PRL was defined as $\geq 30 \mathrm{ng} / \mathrm{mL}$, and normal PRL was defined as $<30 \mathrm{ng} / \mathrm{mL}^{21}$ 


\section{Statistical methods}

Analyses were carried out using the SPSS V.23.0 software. Normally distributed data were presented in the form of mean (SD), and non-normally distributed data were represented by median $(\mathrm{Q} 1, \mathrm{Q} 3)$. The t-tests were performed for data that fit the normal distribution. The Mann-Whitney U test was used to compare non-normally distributed data. Categorical variables were examined by $\chi^{2}$ test. Logistic regression analysis was used to screen the risk factors for PRL increase. All statistical tests were twosided, and $p \leq 0.05$ was considered statistically significant.

\section{RESULTS}

\section{Comparison of demographics and clinical data}

Among the patients taking RIS and OLZ, the proportion of women was significantly greater than that of men (RIS: 82 (52.90\%), $\mathrm{p}=0.009$; OLZ: 47 (81.03\%), $\mathrm{p}<0.001)$, and the average drug dose in the elevated PRL group was greater than that in the normal PRL group (RIS: $4.55(4,6) \mathrm{mg} /$ day vs $3.72(2,4.75) \mathrm{mg} /$ day, $\mathrm{p}<0.001$; OLZ: $14.25(10,20)$ $\mathrm{mg} /$ day vs $11.89(5,16.25) \mathrm{mg} /$ day, $\mathrm{p}=0.021)$. In the RIS group, patients in the elevated PRL group took the drugs for a longer duration than those with normal PRL levels $(0.71(0.11,2.54)$ year vs $0.16(0.01,1.88)$ year, $\mathrm{p}=0.006)$. The study also found that for plasma glucose and lipid values, the elevated PRL group had lower glycaemic values than the normal PRL group in patients taking OLZ, and there was a trend difference in glycaemic values between the two groups in patients taking RIS. In the RIS group, the triglyceride values of patients in the elevated PRL group were lower than those in the normal PRL group. Furthermore, there were no statistically significant differences in age, body mass index, age of onset and illness duration or total cholesterol between RIS and OLZ patients with elevated PRL or the normal groups. The results are shown in table 1 .

\section{Comparison of demographics and clinical data in women}

In the RIS group, the length of medication time in the PRL elevated group was longer than that in the normal PRL group $(0.39(0.08,1.56)$ year vs $0.08(0,1.81)$ year, $\mathrm{p}=0.01)$, and in the OLZ group, there was a trend difference in the medication time between the two groups. Among the patients taking RIS and OLZ, the medication doses of the elevated PRL group were greater than those of the normal PRL group, with significant statistical differences (RIS: 4 (3, 6) $\mathrm{mg} /$ day vs $3(2,4) \mathrm{mg} /$ day, $\mathrm{p}=0.001$; OLZ: $10(10,20)$ $\mathrm{mg} /$ day vs $10(5,15) \mathrm{mg} /$ day, $\mathrm{p}=0.012)$. For plasma glucose and lipids levels, among female patients taking OLZ, there was a statistical difference in fasting plasma glucose between the elevated PRL and the normal PRL groups $(4.94(4.44,5.40) \mathrm{mmol} / \mathrm{L}$ vs $5.21(4.99,5.59) \mathrm{mmol} / \mathrm{L}$, $\mathrm{p}=0.042$ ). Among the RIS group patients, there was a trend difference in triglyceride values. In addition, there were no significant statistical differences in age, illness duration, age of onset, body mass index, total cholesterol or triglyceride. The results are shown in table 2.

\section{Comparison of demographics and clinical data in men}

Among the male patients taking RIS and OLZ, the dosages in the elevated PRL group were higher than those of the normal PRL group (RIS: $4.82(4,6) \mathrm{mg} /$ day vs $4(2,5) \mathrm{mg} /$ day, $\mathrm{p}=0.02$; OLZ: $18.25(11.25,20) \mathrm{mg} /$ day vs $13.52(10,20)$ $\mathrm{mg}$ /day, $\mathrm{p}=0.012)$. The fasting plasma glucose values in the two groups also had statistical differences (RIS: 5.01 (4.60,

Table 1 Comparison of demographic data between patients with elevated or normal PRL levels

\begin{tabular}{|c|c|c|c|c|}
\hline Factors & $\begin{array}{l}\text { Elevated PRL } \\
\text { RIS/OLZ } \\
(n=155 / n=58)\end{array}$ & $\begin{array}{l}\text { Normal PRL } \\
\text { RIS/OLZ } \\
(n=109 / n=117)\end{array}$ & $\begin{array}{l}\text { Statistics } \\
\text { RIS/OLZ }\end{array}$ & $\begin{array}{l}P \text { values } \\
\text { RIS/OLZ }\end{array}$ \\
\hline Age, years & $49(32,58) / 34(27,47)$ & $43(29.25,55) / 38(24,50.25)$ & $U=-1.68 / 0.21$ & $0.093 / 0.834$ \\
\hline Gender/female, number (\%) & 82 (52.90)/47 (81.03) & $40(36.70) / 62(52.99)$ & $\chi^{2}=6.76 / 12.98$ & $0.009^{\star} /<0.001^{*}$ \\
\hline Illness duration, years & $16.50(6.75,31) / 7(1,16)$ & $13(6,24) / 4.32(1,19.82)$ & $U=-1.29 / 0.34$ & $0.197 / 0.733$ \\
\hline Age of onset, years & $26(20.75,30.25) / 25(20.50,33)$ & $25(21,31.5) / 23(19,32.25)$ & $\mathrm{U}=0.42 /-1.14$ & $0.673 / 0.253$ \\
\hline Body mass index, $\mathrm{kg} / \mathrm{m}^{2}$ & $23.48(3.70) / 23.52(4.35)$ & $24.38(4.72) / 22.43(3.46)$ & $t=1.24 / 1.59$ & $0.214 / 0.117$ \\
\hline Dose, mg/day & $4.55(4,6) / 14.25(10,20)$ & $3.72(2,4.75) / 11.89(5,16.25)$ & $\mathrm{U}=-3.73 /-2.31$ & $<0.001^{\star \star} / 0.021^{\star}$ \\
\hline $\begin{array}{l}\text { Fasting plasma glucose, } \\
\mathrm{mmol} / \mathrm{L}\end{array}$ & $5.14(4.75,5.42) / 4.87(4.34,5.30)$ & $5.28(4.80,5.84) / 5.10(4.70,5.43)$ & $\mathrm{U}=1.75 / 2.29$ & $0.080 / 0.022^{*}$ \\
\hline Total cholesterol, mmol/L & $4.51(0.92) / 4.62(0.94)$ & $4.49(0.90) / 4.53(0.93)$ & $t=0.183 / 0.628$ & $0.860 / 0.531$ \\
\hline
\end{tabular}

Normal distribution (mean+SD).

Non-normal distribution (median (Q1, Q3)).

${ }^{*} \mathrm{p} \leq 0.05$

${ }^{* *} \mathrm{p} \leq 0.001$

OLZ, olanzapine; PRL, prolactin; RIS, risperidone. 
Table 2 Comparison of demographic data between female patients with elevated or normal PRL levels

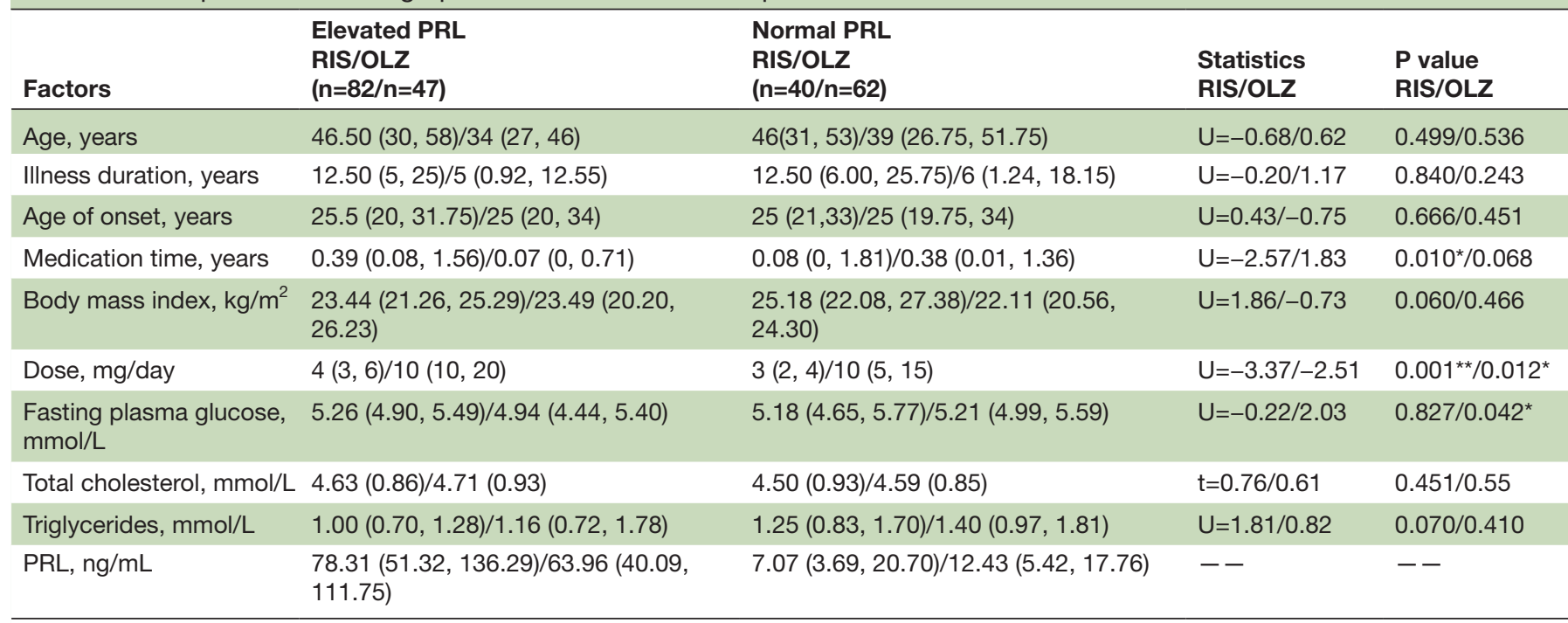

Normal distribution (mean+SD).

Non-normal distribution (median (Q1, Q3)).

${ }^{*} \mathrm{p} \leq 0.05$

${ }^{* *} \mathrm{p} \leq 0.001$

OLZ, olanzapine; PRL, prolactin; RIS, risperidone.

$5.34) \mathrm{mmol} / \mathrm{L}$ vs $5.28(4.84,5.82) \mathrm{mmol} / \mathrm{L}, \mathrm{p}=0.009$; OLZ: $4.60(4.25,4.96) \mathrm{mmol} / \mathrm{L}$ vs $4.94(4.44,5.40) \mathrm{mmol} / \mathrm{L}$, $\mathrm{p}=0.03)$. Furthermore, there were no statistically significant differences in age, medication time, illness duration, age of onset, body mass index or related lipids values. The results are shown in table 3.

Regression analysis of influencing factors of elevated PRL

In this study, regression analysis included 10 variables: age, gender, age of onset, illness duration, medication time, body mass index, dose, fasting plasma glucose, total cholesterol and triglycerides. Logistic regression analysis showed that gender, medication dosage and fasting plasma glucose were significantly associated with elevated PRL. The results are shown in table 4.

Number of patients with elevated and normal PRL levels at different ages

The patients taking RIS or OLZ in this study were divided into the following age groups: $18-30,30-40,40-50,50-60$ and $>60$ years. As shown in figure 2, among the patients taking RIS, the number of patients in the elevated PRL group was larger than that in the normal PRL group, and in the 50-60 age group, the proportion of patients in the

\begin{tabular}{|c|c|c|c|c|}
\hline Factors & $\begin{array}{l}\text { Elevated PRL } \\
\text { RIS/OLZ } \\
(n=73 / n=11)\end{array}$ & $\begin{array}{l}\text { Normal PRL } \\
\text { RIS/OLZ } \\
(n=69 / n=55)\end{array}$ & $\begin{array}{l}\text { Statistics } \\
\text { RIS/OLZ }\end{array}$ & $\begin{array}{l}\text { P value } \\
\text { RIS/OLZ }\end{array}$ \\
\hline Age, years & $51(36,59) / 39(27.25,52.50)$ & $42(29,56.5) / 33(21.25,49.75)$ & $U=-1.76 /-0.79$ & $0.078 / 0.430$ \\
\hline Illness duration, years & $20.89(10.50,35) / 12(5.82,27.88)$ & $13(6,24) / 4.58(1.00,21.88)$ & $U=-1.85 /-1.43$ & $0.664 / 0.152$ \\
\hline Age of onset, years & $26(20,29) / 22.33(20.52,28.25)$ & $25(20,30) / 23(19,30)$ & $U=0.32 /-0.29$ & $0.747 / 0.774$ \\
\hline Medication time, years & $1.24(0.10,2.75) / 0.06(0.003,1.58)$ & $0.66(0.04,2.13) / 0.23(0.01,0.73)$ & $U=-1.74 / 0.35$ & $0.082 / 0.727$ \\
\hline Body mass index, $\mathrm{kg} / \mathrm{m}^{2}$ & $23.42(20.76,25.54) / 23.46(20.57,26.12)$ & $\begin{array}{l}23.84(21.88,25.49) / 22.20(19.85, \\
24.08)\end{array}$ & $\mathrm{U}=0.37 / 1.13$ & $0.713 / 0.199$ \\
\hline Dose, mg/day & $4.82(4,6) / 18.25(11.25,20)$ & $4(2,5) / 13.52(10,20)$ & $U=-2.33 / 2.52$ & $0.020^{*} / 0.012^{*}$ \\
\hline $\begin{array}{l}\text { Fasting plasma glucose, } \\
\mathrm{mmol} / \mathrm{L}\end{array}$ & $5.01(4.60,5.34) / 4.60(4.25,4.96)$ & $5.28(4.84,5.82) / 4.94(4.44,5.40)$ & $\mathrm{U}=2.62 / 2.15$ & $0.009^{*} / 0.03^{*}$ \\
\hline Total cholesterol, $\mathrm{mmol} / \mathrm{L}$ & $4.38(0.97) / 4.27(0.90)$ & $4.47(0.91) / 4.46(0.94)$ & $\mathrm{t}=-0.58 /-0.63$ & $0.556 / 0.529$ \\
\hline Triglycerides, mmol/L & $1.27(0.93,1.57) / 1.43(0.72,2.69)$ & $1.46(0.99,1.97) / 1.35(0.91,1.99)$ & $U=1.79 / 0.36$ & $0.073 / 0.720$ \\
\hline $\mathrm{PRL}, \mathrm{ng} / \mathrm{mL}$ & $47.34(36.18,60.59) / 42.12(32.49,56.13)$ & $12.70(2.64,18.03) / 12.55(2.76,18.31)$ & -- & -- \\
\hline
\end{tabular}

Normal distribution (mean+SD).

Non-normal distribution (median (Q1, Q3)).

${ }^{*} \mathrm{p} \leq 0.05$

OLZ, olanzapine; PRL, prolactin; RIS, risperidone. 
Table 4 Analysis of risk factors of elevated PRL

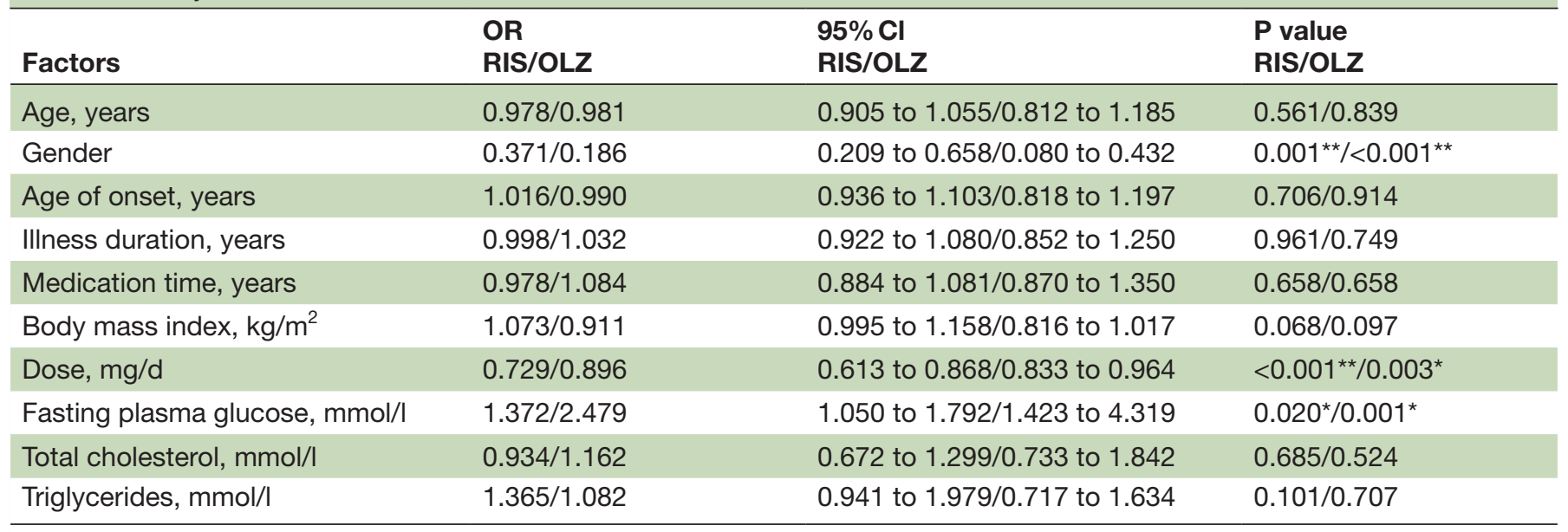

${ }^{*} \mathrm{p} \leq 0.05$

${ }^{* *} \mathrm{p} \leq 0.001$

OLZ, olanzapine; PRL, prolactin; RIS, risperidone.

elevated PRL group was larger. In the OLZ group, the number of patients with elevated PRL was lower than that of the normal PRL group.

\section{DISCUSSION}

\section{Main findings}

With the widespread use of RIS and OLZ, their effects on PRL levels have received increasing attention. Elevated PRL levels can lead to breast overflow in women and breast development in men and can inhibit progesterone and oestrogen, causing delayed menstruation, amenorrhoea, infertility and endometrial cancer. ${ }^{22}$ In addition, PRL can stimulate the proliferation of osteoclast precursors, reduce bone density, increase the risk of fractures and increase the secretion of interleukin- 6 by inhibiting oestradiol levels. ${ }^{23}$ These consequences can seriously affect the quality of life.

This study found that gender played a role in the effects of RIS and OLZ on serum PRL. The proportion of female patients in the elevated PRL group was larger. Logistic regression analysis also showed that gender was a risk factor for elevated PRL. This finding was consistent

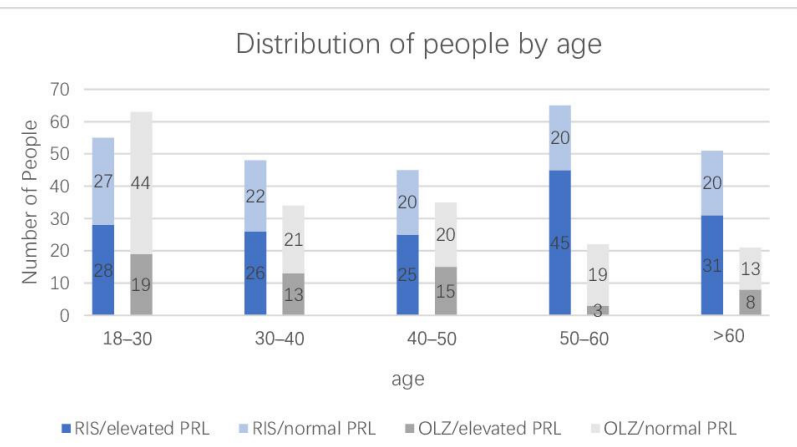

Figure 2 Number of patients with elevated and normal PRL levels at different ages. OLZ, olanzapine; PRL, prolactin; RIS, risperidone. with some previous reports in the literature. Kearns et $a l^{24}$ found that the PRL level of women was significantly higher than that of men among those taking RIS, which was consistent with the conclusions of the study by Bushe and Shaw. ${ }^{25}$ Moreover, the results of an epidemiological investigation in China showed that gender is one of the independent factors influencing the increase in PRL levels caused by antipsychotic use. ${ }^{26}$ Therefore, combined with the results of this study, there is a greater likelihood of female patients taking RIS or OLZ being affected by the increased PRL levels, and steps to prevent female-specific PRL increase during clinical use should be explored.

This study also found a correlation between the doses of RIS and OLZ and elevated PRL. Other studies have reported that the level of PRL decreased significantly in patients with schizophrenia after a reduction in the dose of medication. ${ }^{7}$ There are also reports in the literature indicating that the level of elevated PRL is dose dependent: the larger the dose, the higher the level of PRL. ${ }^{4}$ This suggests that antipsychotic dosage may be an important predictor of hyperprolactinaemia. Decreasing the dose of antipsychotic drugs is feasible for patients with schizophrenia in order to reduce PRL.

The results showed that for patients taking RIS or OLZ, the elevated PRL group had lower plasma glucose levels than the normal PRL group. Previous studies have found that low-dose PRL injections enhanced insulin secretion through glucokinase and glucose transporter 2 in diabetic rats. ${ }^{27}$ In recent years, attention has focused on the relationship between PRL, diabetes and glucose regulation in China. The relationship between PRL and glucose regulation has been investigated in a large sample of men and women based in Shanghai. Investigators found that physiologically high PRL levels were associated with a lower prevalence of diabetes in both men and postmenopausal women. ${ }^{11}$ In addition, studies have found that in diabetic rats, a low dose of PRL ( $25 \mu \mathrm{g} / \mathrm{kg}$ bw/12 hours) enhances 
glucose-stimulated insulin secretion through glucokinase and glucose transporter 2 induction and reduces plasma glucose. However, the high-dose PRL injection exacerbated systemic and hepatic insulin resistance. ${ }^{27}$ This suggests that the degree of elevated PRL levels may have different effects on glucose metabolism. In this study, PRL levels in patients with schizophrenia after taking RIS and OLZ were generally below $100 \mu \mathrm{g} / \mathrm{mL}$, and high serum PRL was associated with a favourable glucose metabolic profile. This suggests a possible association between PRL levels and $\beta$-cell function in patients with schizophrenia after taking RIS and OLZ. Furthermore, in our study, female patients taking RIS had a slight decrease in plasma glucose level in the elevated PRL group, but there were no statistical differences between the two groups. However, in male patients, there was a significant statistical difference in plasma glucose between the two groups. Perhaps because PRL secretion varies at different stages of the menstrual cycle, there were more confounding factors affecting PRL secretion in female patients. ${ }^{28}$

This study is a real-world, multicentre clinical trial. In the real world, the influencing factors of patients' elevated PRL levels were analysed. Because previous studies have shown a correlation between PRL and plasma glucose and lipids, this study included plasma glucose and lipids in the analysis of PRL levels in Chinese patients taking antipsychotic drugs. The results showed that the plasma glucose levels of the elevated PRL group were lower than those of the group with normal PRL levels. Thus, there may be a correlation between PRL levels and plasma glucose.

\section{LIMITATIONS}

This study was based on cross-sectional data from a realworld study, so it was impossible to demonstrate trends and correlations of dose, plasma glucose and PRL levels in patients with schizophrenia over a long period. In addition, some patients in this study also took other antipsychotic drugs while taking RIS and OLZ, and PRL levels could have been affected by the other drugs. Prospective studies are needed to clarify the relationship and role of plasma glucose and PRL. In the future, longitudinal follow-up studies will be conducted to further expand this study and explore the related factors affecting elevated PRL caused by the use of antipsychotic drugs. Furthermore, because the degree of PRL elevation and its corresponding effect on glucose metabolism may be different, further research is needed to analyse and find the critical value of the effect of PRL on plasma glucose.

\section{IMPLICATIONS}

In summary, this study has found that in patients with schizophrenia taking RIS and OLZ, gender and dose were significantly correlated with the PRL value. Moreover, the blood glucose level of the group with elevated PRL was lower than that of the group with normal PRL.
Contributors SH and WJY are responsible for the statistical analysis and the writing of the paper. XW, LZ and NZ are responsible for collecting patient information. GL and YFS contributed to the research. HL is responsible for the scientific design of the study.

Funding The authors have not declared a specific grant for this research from any funding agency in the public, commercial or not-for-profit sectors.

Competing interests None declared.

Patient consent for publication Not required.

Ethics approval The study was organised by the Shanghai Mental Health Center, reviewed and approved by its ethics committee and assigned to the International Clinical Trials Registry Platform.

Provenance and peer review Not commissioned; externally peer reviewed.

Data availability statement Data are available on reasonable request. Multicenter Real World Clinical Study of Long-term Outcomes for Schizophrenia by Atypical Antipsychotic Treatment in China.

Open access This is an open access article distributed in accordance with the Creative Commons Attribution Non Commercial (CC BY-NC 4.0) license, which permits others to distribute, remix, adapt, build upon this work non-commercially, and license their derivative works on different terms, provided the original work is properly cited, appropriate credit is given, any changes made indicated, and the use is non-commercial. See: http://creativecommons.org/licenses/by-nc/4.0/.

\section{REFERENCES}

1 Sullivan PF, Kendler KS, Neale MC. Schizophrenia as a complex trait: evidence from a meta-analysis of twin studies. Arch Gen Psychiatry 2003;60:1187-92.

2 Zhang C, Mao Y, Song L. Precise treatments for schizophrenia: where is the way forward? Gen Psychiatr 2018;31:e000002.

3 Fitzgerald P, Dinan TG. Prolactin and dopamine: what is the connection? A review article. J Psychopharmacol 2008;22:12-19.

4 Wenjuan Y, Liping Z. Mechanisms of anti-psychotics inducing high prolactin [J]. Journal of psychiatry 2011;24:235-7.

5 Kinon BJ, Gilmore JA, Liu H, et al. Prevalence of hyperprolactinemia in schizophrenic patients treated with conventional antipsychotic medications or risperidone. Psychoneuroendocrinology 2003;28:55-68.

6 Crespo-Facorro B, Ortiz-Garcia de la Foz V, Suarez-Pinilla P, et al. Effects of aripiprazole, quetiapine and ziprasidone on plasma prolactin levels in individuals with first episode nonaffective psychosis: analysis of a randomized open-label 1year study. Schizophr Res 2017;189:134-41.

7 Besnard I, Auclair V, Callery G, et al. [Antipsychotic-drug-induced hyperprolactinemia: physiopathology, clinical features and guidance]. Encephale 2014;40:86-94.

8 Haas M, Eerdekens M, Kushner S, et al. Efficacy, safety and tolerability of two dosing regimens in adolescent schizophrenia: double-blind study. Br J Psychiatry 2009;194:158-64.

9 Graff-Guerrero A, Rajji TK, Mulsant BH, et al. Evaluation of antipsychotic dose reduction in late-life schizophrenia: a prospective dopamine D2/3 receptor occupancy study. JAMA Psychiatry 2015;72:927-34.

10 Huang $\mathrm{Y}$, Chang $\mathrm{Y}$. Regulation of pancreatic islet beta-cell mass by growth factor and hormone signaling. Prog Mol Biol Trans/ Sci 2014;121:321-49.

11 Wang T, Lu J, Xu Y, et al. Circulating prolactin associates with diabetes and impaired glucose regulation: a population-based study. Diabetes Care 2013;36:1974-80.

12 Huang C, Snider F, Cross JC. Prolactin receptor is required for normal glucose homeostasis and modulation of beta-cell mass during pregnancy. Endocrinology 2009;150:1618-26.

13 Sorenson RL, Brelje TC. Prolactin receptors are critical to the adaptation of islets to pregnancy. Endocrinology 2009;150:1566-9.

14 Brandebourg T, Hugo E, Ben-Jonathan N. Adipocyte prolactin: regulation of release and putative functions. Diabetes Obes Metab 2007;9:464-76.

15 Ling C, Svensson L, Odén B, et al. Identification of functional prolactin (PRL) receptor gene expression: PRL inhibits lipoprotein lipase activity in human white adipose tissue. $J$ Clin Endocrinol Metab 2003;88:1804-8.

16 Nilsson L, Binart N, Bohlooly-Y M, et al. Prolactin and growth hormone regulate adiponectin secretion and receptor expression in adipose tissue. Biochem Biophys Res Commun 2005;331:1120-6. 
17 Ozisik H, Suner A, Cetinkalp S. Prolactin effect on blood glucose and insülin in breastfeeding women. Diabetes Metab Syndr 2019;13:1765-7.

18 Mauri MC, Paletta S, Maffini M, et al. Clinical pharmacology of atypical antipsychotics: an update. Excli J 2014;13:1163-91.

19 Xia L, Li W-Z, Liu H-Z, et al. Olanzapine versus risperidone in children and adolescents with psychosis: a meta-analysis of randomized controlled trials. J Child Adolesc Psychopharmacol 2018;28:244-51.

20 Huhn M, Nikolakopoulou A, Schneider-Thoma J, et al. Comparative efficacy and tolerability of 32 oral antipsychotics for the acute treatment of adults with multi-episode schizophrenia: a systematic review and network meta-analysis. Lancet 2019;394:939-51.

21 Serri O, Chik CL, Ur E, et al. Diagnosis and management of hyperprolactinemia. CMAJ 2003;169:575-81.

22 Riecher-Rössler A. Oestrogens, prolactin, hypothalamic-pituitarygonadal axis, and schizophrenic psychoses. Lancet Psychiatry 2017;4:63-72.

23 Lee JS, LaCroix AZ, Wu L, et al. Associations of serum sex hormone-binding globulin and sex hormone concentrations with hip fracture risk in postmenopausal women. $J$ Clin Endocrinol Metab 2008:93:1796-803.

24 Kearns AE, Goff DC, Hayden DL, et al. Risperidone-associated hyperprolactinemia. Endocr Pract 2000;6:425-9.

25 Bushe C, Shaw M. Prevalence of hyperprolactinaemia in a naturalistic cohort of schizophrenia and bipolar outpatients during treatment with typical and atypical antipsychotics. $J$ Psychopharmacol 2007;21:768-73.

26 Yunai S, Li Qian SL, et al. Analysis of gender differences in drug treatment of schizophrenic patients in ten provinces and cities of China in 2012 [J]. Chinese Journal of Psychiatry 2016;49:367-72.

27 Park S, Kim DS, Daily JW, et al. Serum prolactin concentrations determine whether they improve or impair $\beta$-cell function and insulin sensitivity in diabetic rats. Diabetes Metab Res Rev 2011;27:564-74.

28 Caufriez A, Leproult R, L'Hermite-Balériaux M, et al. A potential role of endogenous progesterone in modulation of $\mathrm{GH}$, prolactin and thyrotrophin secretion during normal menstrual cycle. Clin Endocrinol 2009;71:535-42.

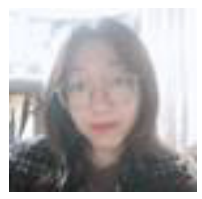

Sidi He graduated from Bengbu Medical college in 2017, and she is currently a postgraduate student of Shanghai Mental Health Center, Medical college in Shanghai Jiao Tong University, Shanghai, China. Her research interest includes increased prolactin levels in patients with schizophrenia. 